OPEN ACCESS

Edited by:

Nihal Ahmad,

University of Wisconsin-Madison,

United States

Reviewed by:

Gagan Chhabra,

University of Wisconsin-Madison,

United States

Jeffrey Winters,

Mayo Clinic, United States

${ }^{*}$ Correspondence:

David Rafei-Shamsabadi

david.rafei-shamsabadi@uniklinik-

freiburg.de

Specialty section:

This article was submitted to

Skin Cancer,

a section of the journal

Frontiers in Oncology

Received: 25 January 2021

Accepted: 24 March 2021

Published: 13 April 2021

Citation:

Rafei-Shamsabadi D, Schneider J,

Trefzer L, Technau-Hafsi K, Meiss F

and Ness T (2021) Case Report:

Blurred Vision and Eruptive Nevi -

Bilateral Diffuse Uveal Melanocytic

Proliferation With Mucocutaneous Involvement in a Lung Cancer Patient.

Front. Oncol. 11:658407.

doi: 10.3389/fonc.2021.658407

\section{Case Report: Blurred Vision and Eruptive Nevi - Bilateral Diffuse Uveal Melanocytic Proliferation With Mucocutaneous Involvement in a Lung Cancer Patient}

\author{
David Rafei-Shamsabadi ${ }^{1 *}$, Johanna Schneider ${ }^{2}$, Laura Trefzer $^{1}$, Kristin Technau-Hafsi $^{1}$, \\ Frank Meiss ${ }^{1}$ and Thomas Ness ${ }^{3}$ \\ 1 Department of Dermatology and Venereology, Medical Center - University of Freiburg, Faculty of Medicine, University of \\ Freiburg, Freiburg, Germany, ${ }^{2}$ Department of Medicine IV, Medical Center - University of Freiburg, Faculty of Medicine, \\ University of Freiburg, Freiburg, Germany, ${ }^{3}$ Eye Center, University Hospital Freiburg, Faculty of Medicine, University of \\ Freiburg, Freiburg, Germany
}

We describe a case of a 65-year old patient presenting with unusual mucocutaneous melanocytic proliferations of a Bilateral Diffuse Uveal Melanocytic Proliferation (BDUMP) imitating a multifocal melanoma in situ, which improved dramatically after plasmapheresis. The patient first presented at the dermatology department due to rapidly evolving brown and black macules on the glans penis. Further skin involvement of the perineal and perianal region, mamillae and oral mucosa was stated. Histology from a penile biopsy was compatible with a melanoma in situ. Due to the distribution pattern and elevated serum tumor marker S100B, metastatic melanoma was considered. Staging examinations using PET-CT scan however, revealed a lung tumor, later confirmed as a Non-small-cell lung cancer (NSCLC). Primary radio chemotherapy was initiated to treat NSCLC. Shortly after initiation of radio chemotherapy the patient developed massive vision impairment and a NSCLC-associated BDUMP was diagnosed which led to the correct classification of melanocytic skin lesions as mucocutaneous BDUMP manifestation. Plasmapheresis was started resulting in a rapid improvement of vision starting ten days after the first plasmapheresis. In contrast skin manifestations started to disappear with a marked delay 4 months after the last plasmapheresis cycle. This case highlights the importance of memorizing multiple rapidly progressing melanocytic skin and/or mucous membrane spots together with visual impairment as a possible paraneoplastic BDUMP that needs a fundamentally different therapeutic approach compared to multifocal melanoma in situ.

What is already known about this topic? Bilateral Diffuse Uveal Melanocytic Proliferation (BDUMP) is a paraneoplastic syndrome with melanocytic uveal proliferation leading to vision impairment. Extraocular manifestation is rare, mainly affect the subepidermal compartment and is hard to treat. Plasmapheresis has been shown to be an effective treatment mainly for vision improvement in some but not all cases. 
What does this study add? Our BDUMP case with widespread skin and mucosal involvement initially mimicked a multifocal melanoma in situ and showed an excellent treatment response to plasmapheresis. Improvement of mucocutaneous lesions has not been documented well in the literature so far. We show a more than one year lasting follow up still underlining the beneficial effect of plasmapheresis in this case. In-vitro data supports the hypothesis that plasma exchange eliminates a "Cultured melanocyte elongation and proliferation (CMEP)" factor out of patient blood leading to decreased melanocyte proliferation shown numerically in-vitro and clinically in-vivo. Our case clearly indicates that before establishing a definite diagnosis and therapy in patients with rapidly evolving melanocytic skin and/or mucosal lesions BDUMP mimicking multifocal melanoma in situ should be considered making a thorough diagnostic workup mandatory.

Keywords: bilateral diffuse uveal melanocytic proliferation (BDUMP), skin involvement, melanoma, plasmapheresis, non-small-cell lung cancer (NSCLC)

\section{INTRODUCTION}

We present a case of a Non-small-cell lung cancer (NSCLC)associated Bilateral Diffuse Uveal Melanocytic Proliferation (BDUMP), which mimicked a multifocal melanoma in situ with diffuse skin and mucous membrane involvement showing epidermal proliferation only. Impaired vision dramatically improved shortly after start of plasmapheresis treatment whereas mucocutaneous lesions showed regression with profoundly delayed dynamics. The patient gave written consent for publishing clinical pictures and data gained from scientific usage of skin biopsies and plasma.

\section{PATIENT INFORMATION}

A 65-year old male patient was sent to our outpatient department with rapidly growing black macules on the penis (Figures 1A, B). He reported no clinical symptoms at that time but was concerned about the increasing extent of these spots. The patient had a history of larynx carcinoma with neck dissection and adjuvant radiotherapy and squamous cell carcinoma of the left conjunctiva also excised with clear margins. Both tumors occurred more than 10 years ago. The patient had smoked for several years but had quit 12 years prior. The family history was unremarkable concerning malignant diseases.

\section{CLINICAL FINDINGS}

At first visit, multiple black, homogenous spots some of them discretely sublime on the glans penis, dorsum penis and scrotum were evident (Figures 1A, B). Dermoscopy of these lesions showed homogeneous dark-brown and black spots largely lacking net-like structures (Figure 1C). No other pathologic skin lesions were identified. No pathological lymph nodes were palpable.

\section{DIAGNOSTIC ASSESSMENT}

Histology of biopsies from penis and scrotum revealed multifocal melanoma in situ with atypical melanocytes showing a focal pagetoid infiltration of the epidermis and strong junctional melanin pigmentation with marked pigmentary incontinence (Figure 1D). Intraepidermal melanocytes expressed SOX10, HMB45, und S100 (Figures 1E-G). The serum S100B level, a tumor marker for malignant melanoma, was increased $(0.353 \mu \mathrm{g} / \mathrm{l}$, norm $<0.105 \mu \mathrm{g} / \mathrm{l}$ ) while lactate dehydrogenase (LDH) was normal. The considered diagnosis was a metastatic melanoma with multiple skin lesions (epidermotropic metastases) with unknown primary. A PET-CT scan revealed a suspicious lesion in the lower lobe of the left lung and pathological lymph nodes in the corresponding hilus region (Figure $\mathbf{1 H}$ ). No further suspicious lesions were detected. An MRI of the brain was normal. Endobronchial ultrasound (EBUS) guided biopsies detected a pleomorphic adenocarcinoma of the lung (NSCLC) but no signs of malignant melanoma.

\section{THERAPEUTIC INTERVENTION}

Under the initial assumption of two malignancies, we first planned a curative resection of the multifocal melanoma in situ followed by an operation and subsequent adjuvant therapy of the NSCLC. Meanwhile the patient had developed further melanocytic lesions on the oral mucosa and the perianal region (Figure 2A). Thus, resection was abandoned. Instead, primary radio chemotherapy (cumulative dose of $66 \mathrm{~Gy}+$ Cisplatin and Vinorelbine) of the NSCLC was started. During radiochemotherapy, the patient experienced a sudden impairment of vision on both eyes with a complete vision loss. Funduscopic and optical coherence tomography (OCT) showed subretinal melanoma like lesions with a massive serous retinal detachment in both eyes (Figures 3A, B). Based on these findings paraneoplastic Bilateral Diffuse Uveal Melanocytic Proliferation (BDUMP) was diagnosed and pigmentary skin lesions were 


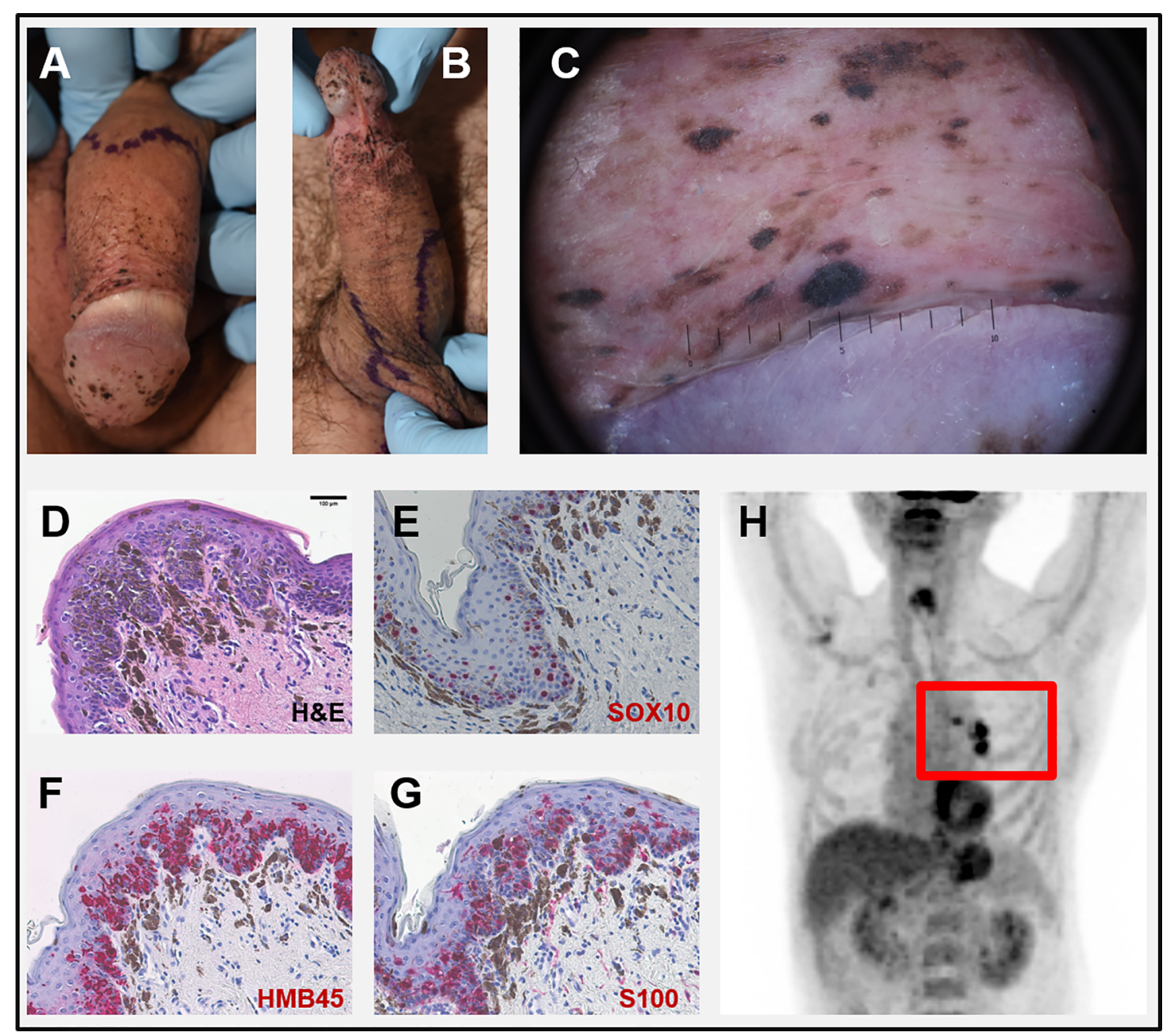

FIGURE 1 | (A, B) Clinical pictures at first visit: multiple slightly palpable black spots on penis and scrotum. (C) Dermoscopy of a corresponding lesion of the glans penis: homogeneous dark-brown and black spots largely lacking net-like structures. (D-G) Microscopic pictures of a biopsy taken from the penis. (D) H\&E-staining showing atypical melanocytes with focal pagetoid infiltration of the epidermis and strong junctional melanocytic pigmentation with pigmentary incontinence. Intraepidermal melanocytes strongly express SOX10 (E), HMB45 (F), and S100 (G). (H) PET-CT scan with highly suspicious lymph nodes in the left hilus region (red rectangle). Scale bar in $\mathrm{D}=100 \mu \mathrm{m}$.

reevaluated as mucocutaneous manifestation of BDUMP. Initially, high dose systemic steroids for one week did not lead to any improvement of vision and thus plasmapheresis (11 times over a period of 3 weeks with exchange of one plasma volume per procedure) was started.

\section{FOLLOW-UP AND OUTCOMES}

After seven cycles of plasmapheresis cycles (10th day after start of treatment), the patient experienced a rapid improvement of vision. Subsequently, retinal detachment declined as well and vision turned back to normal (Figure 3B). The melanocytic skin and mucous membrane lesions however initially remained stable. Four months after the last plasmapheresis cycle melanocytic lesions started to decrease as well (Figure 2B). The NSCLC showed stable disease under radio chemotherapy and therapy was switched to a PD-L1-inhibitor (Durvalumab) resulting in a partial response (for a timeline summarizing the relevant data from the episode of care see Figure 3C).

\section{IN-VITRO DATA}

Patient plasma obtained before and after plasmapheresis was incubated together with neonatal human epidermal melanocytes for one week in-vitro. Compared to healthy control plasma and medium alone, patient plasma lead to a concentration dependent significant increase in melanocyte cell numbers, melanin pigmentation and elongation of dendrites (Figures 4A, B). 


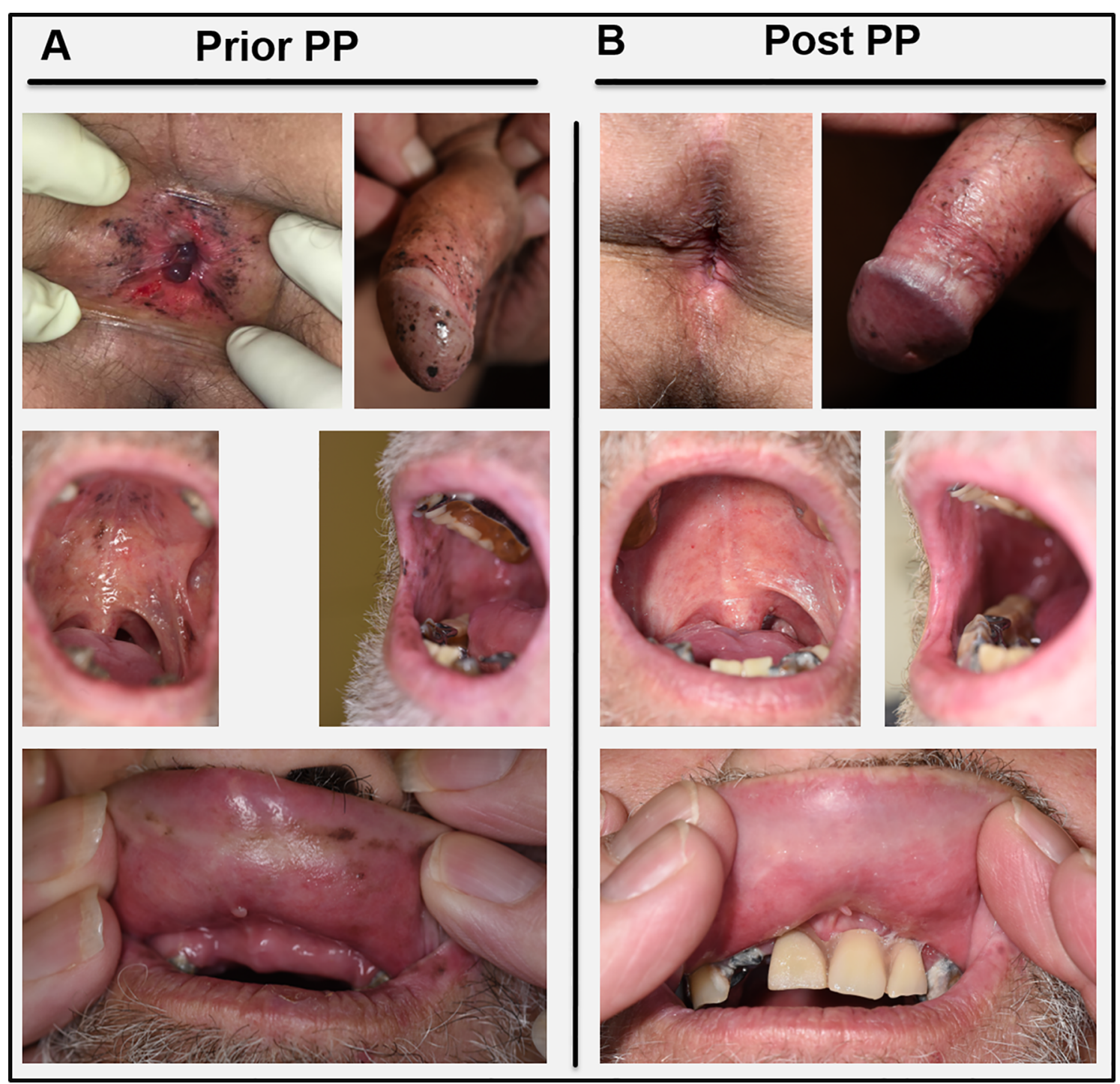

FIGURE 2 | (A) Skin and mucous membrane manifestations of BDUMP before initiation of plasmapheresis (Prior PP). (B) Clinical presentation four months after the last PP cycle (Post PP) with profound decrease of melanocytic proliferations especially on the oral mucous membranes and in the perianal region. Pictures in the lowest panel were taken without (left) and with (right) the patients' dental prosthesis.

This effect decreased after the fourth cycle of plasmapheresis and further diminished 4 months after the last treatment. However, the number of proliferating melanocytes was still higher compared to the controls (Figure 4A).

\section{DISCUSSION}

In the literature, BDUMP is described as a paraneoplastic disease accompanying predominantly tumors of the female urogenital tract and the lung. Mucocutaneous manifestations of BDUMP have been described in around $10-15 \%$ of cases $(1,2)$. As a possible pathogenic factor secreted by tumor cells the so called
"Cultured melanocyte elongation and proliferation (CMEP)" factor has been discussed which presumably belongs to the IgG fraction of serum (3). Possible candidates promoting melanocytic growth comprise $\alpha$-melanocyte-stimulating hormone $(\alpha-\mathrm{MSH})$, adrenocorticotropic hormone (ACTH), fibroblast growth factor (FGF), hepatocyte growth factor (HGF), granulocyte macrophage colony-stimulating factor (GM-CSF) and endothelins $(4,5)$.

Therapeutic options for BDUMP besides treatment of the underlying tumor are scarce. Systemic steroids were effective in some cases (1). However, our patient did not experience any improvement of vision after one week of high dose systemic steroids. Plasmapheresis has been shown to reduce the subretinal fluid and to improve vision (6-9). This holds true for our 


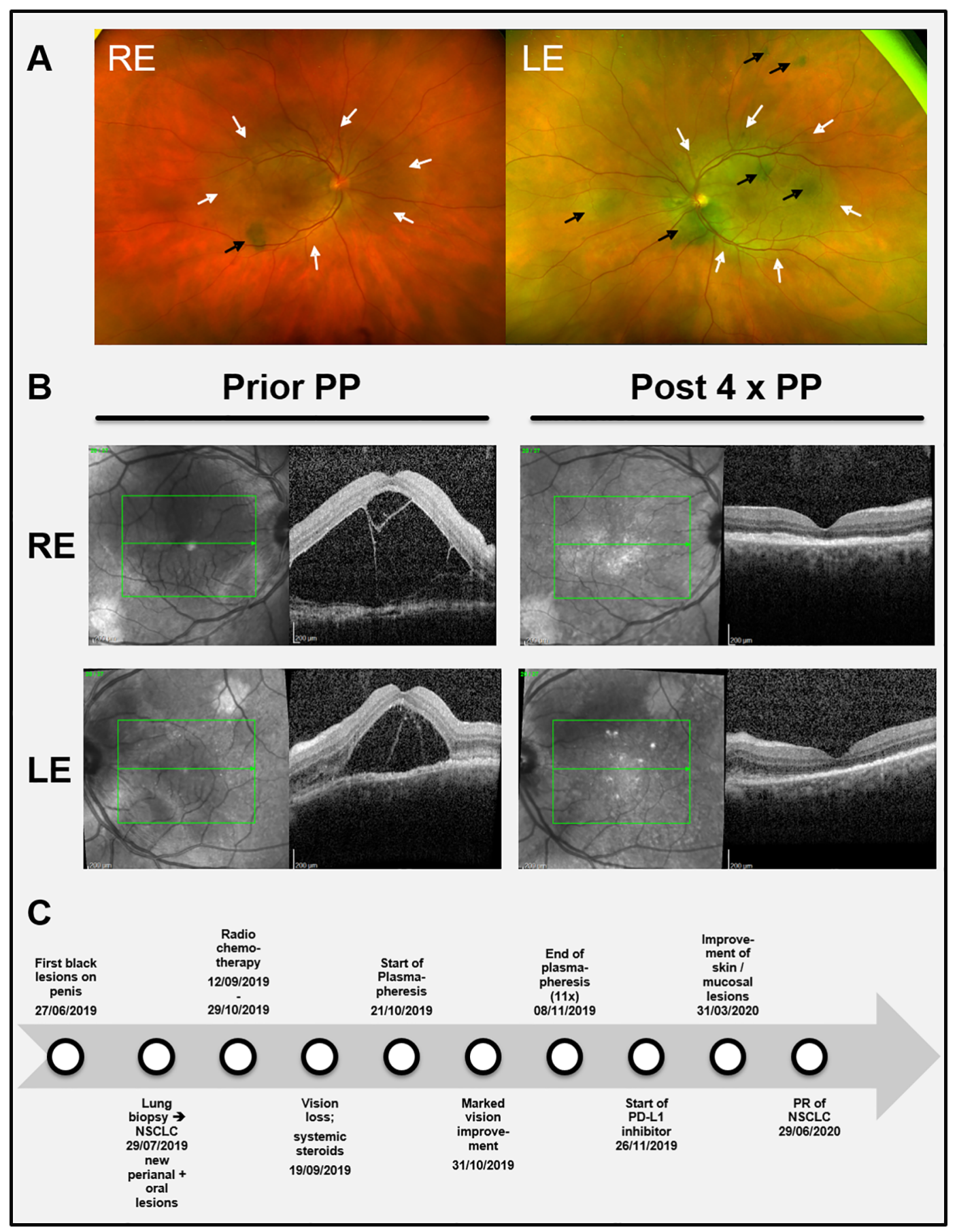

FIGURE 3 | (A) Color funduscopic pictures showing the initial ocular findings. Black arrows: subretinal melanocytic proliferations; white arrows: area of serous retinal detachment. (B) (left part): Black and white funduscopic and optical coherence tomography (OCT) images of the right eye (RE) and the left eye (LE) before start of plasmapheresis (Prior PP) when visual impairment was most prominent leading to total blindness. (B right part) Corresponding images after four cycles of plasmapheresis (Post $4 \times$ PP) showing a remarkable reduction of the subretinal edema and the retinal detachment, respectively. (C) Timeline summarizing the relevant data from the episode of care. NSCLC $=$ Non-small-cell lung cancer. PD-L1 = Programmed death-ligand 1.

case since retinal detachment and loss of vision was completely reversed already after eleven cycles of plasmapheresis.

Pretreatment plasma dramatically increased melanocyte proliferation in vitro. Plasmapheresis via a column with a pore size of $0.3 \mu \mathrm{m}$ lead to a fast and long-lasting remission of melanocytic proliferation starting in the uveal area. Furthermore, with the apheresis devices used the IgG fraction of the serum is being reduced significantly as shown by Hafer 


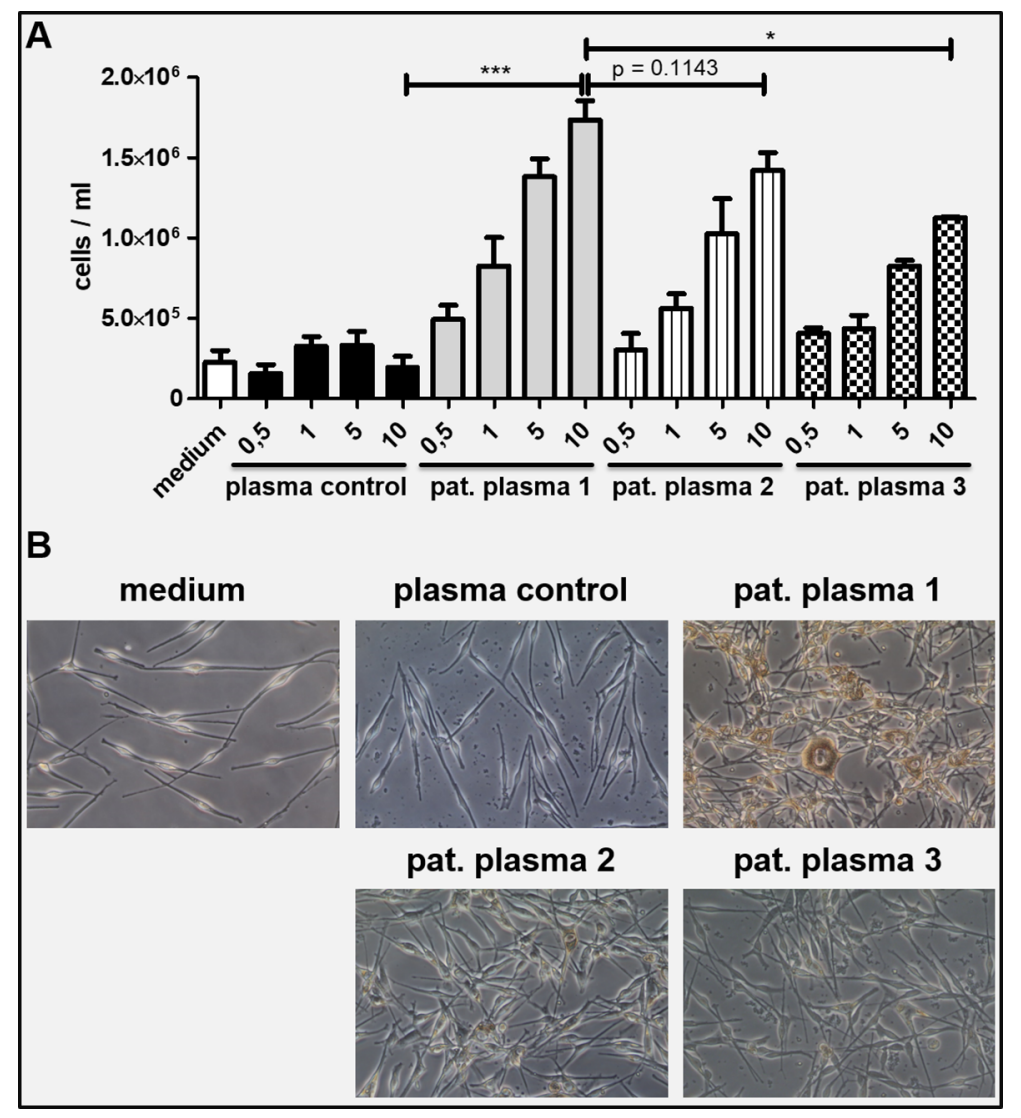

FIGURE 4 | (A) Neonatal human melanocytes were incubated with patient plasma under daily medium changes for 7 days. A concentration dependent increase of total cell numbers was observed which was not seen with healthy control plasma and medium control. The effect diminished with ongoing plasmapheresis ( 1 = plasma from first plasmapheresis; 2 = plasma from second plasmapheresis; 3 = plasma taken 4 months after last plasmapheresis; $x$-axis showing percentage of plasma volume diluted in culture medium. (B) Microscopic pictures of the melanocyte cultures on day 7 (10\% plasma conditions): Melanocytes incubated with 10\% patient plasma 1 show markedly increased proliferation, more dendrites and higher melanin production compared to plasma control and to a lesser extent to patient plasma from second plasmapheresis and 4 months after last plasmapheresis, respectively. An unpaired, two-tailed t test was performed to compare conditions with $10 \%$ plasma volume. Conditions were run at least in duplicates. Values are shown as mean $\pm \mathrm{SD}$. ${ }^{*} \mathrm{P} \leq 0.05,{ }^{* * *} \mathrm{P} \leq 0.001$.

et al. (10). Thus, it is tempting to speculate that the concentration of CMEP factor has been successfully decreased by this treatment as seen by the clinical benefit and the reduced capacity of the patient plasma to induce melanocyte proliferation in vitro. The delayed onset of treatment response of mucocutaneous lesions compared to uveal lesions could be explained by the superior blood supply in the uvea. In contrast to other cases with skin involvement $(11)$, our and other BDUMP cases $(5,12)$ showed solely intraepidermal melanocytic proliferation where blood vessels are missing and growth factors act via diffusion to the epidermal compartment and hence the effect of reducing CMEP by plasmapheresis may be delayed in intraepidermal variants. However, since we could not take samples from all skin and mucosal lesions we therefore cannot fully exclude that dermal proliferation was present at other sites in our patient. According to the hypothesis of Gass et al. (12), the CMEP factor may act on preexisting nevus cells in the dermal and/or epidermal compartment leading to BDUMP with multifocal pigmented lesions.
Although skin lesions including the genital area and mucosal involvement have been described in a few BDUMP cases $(2,5$, $11,13,14)$, our case is unique regarding the extent of skin and mucous membrane involvement, the solely epidermal proliferation and the reported response-pattern of these lesions to plasmapheresis. Little is known about the long-term development of the melanocytic lesions under therapy. In our case, plasma exchange showed immediate improvement of vision and a nearly complete reduction of mucocutaneous melanocytic lesions, which continues to last up to one year and started long before treatment response of the underlying NSCLC could be achieved by systemic therapy.

We cannot rule out, that radio chemotherapy and/or immunotherapy of the NSCLC had an additive effect on the improvement of the mucocutaneous BDUMP lesions. The imminent improvement of vision however underlines the antiproliferative effect of plasmapheresis.

Before establishing a definite diagnosis and therapy in patients with rapidly evolving melanocytic skin and/or mucosal 
lesions BDUMP mimicking multifocal melanoma in situ should be considered. Thorough clinical and radiological diagnostics including fundoscopy should be performed. Finally, plasmapheresis is a well-tolerated treatment option for BDUMP, which can be performed in parallel to systemic tumor treatments or radiotherapy. A long-term follow up is needed if possible to assess the therapeutic effect of plasmapheresis on mucocutaneous BDUMP lesions.

\section{PATIENT PERSPECTIVE}

"I was scared when I suddenly lost my vision. I had to trust the physicians to fix that. Receiving this "blood cleaning" while not seeing anything was weird but I was so glad when I could see again after some days. I hope the lung tumor will continue to shrink as well.” (Patients' quote).

\section{DATA AVAILABILITY STATEMENT}

The raw data supporting the conclusions of this article will be made available by the authors, without undue reservation.

\section{ETHICS STATEMENT}

Written informed consent was obtained from the individual(s) for the publication of any potentially identifiable images or data included in this article.

\section{AUTHOR CONTRIBUTIONS}

DR-S provided the leading contribution in: conceptualization, data curation, formal analysis, investigation, methodology,

\section{REFERENCES}

1. Klemp K, Kiilgaard JF, Heegaard S, Nørgaard T, Andersen MK, Prause JU. Bilateral diffuse uveal melanocytic proliferation: Case report and literature review. Acta Ophthalmol (2017) 95:439-45. doi: 10.1111/aos.13481

2. O'Neal KD, Butnor KJ, Perkinson KR, Proia AD. Bilateral diffuse uveal melanocytic proliferation associated with pancreatic carcinoma: a case report and literature review of this paraneoplastic syndrome. Survey Ophthalmol (2003) 48:613-25. doi: 10.1016/j.survophthal.2003.08.005

3. Miles SL, Niles RM, Pittock S, Vile R, Davies J, Winters JL, et al. A factor found in the IgG fraction of serum of patients with paraneoplastic bilateral diffuse uveal melanocytic proliferation causes proliferation of cultured human melanocytes. Retina (Philadelphia Pa) (2012) 32:1959-66. doi: 10.1097/ IAE.0b013e3182618bab

4. Halaban R. The regulation of normal melanocyte proliferation. Pigment Cell Res (2000) 13:4-14. doi: 10.1034/j.1600-0749.2000.130103.x

5. Tada K-I, Higashi Y, Uchida Y, Kawai K, Nakao K, Misono T, et al. Bilateral diffuse uveal melanocytic proliferation with mucocutaneous pigmentation. J Dermatol (2015) 42:1183-5. doi: 10.1111/1346-8138.12982

6. Jansen JCG, Van Calster J, Pulido JS, Miles SL, Vile RG, Van Bergen T, et al. Early diagnosis and successful treatment of paraneoplastic melanocytic project administration, resources, software, visualization, writing-original draft, writing-review \& editing. JS supported in data curation, investigation, methodology, resources, writingreview \& editing. LT supported in data curation, investigation, writing-review \& editing. KT-H supported in data curation, investigation, resources, writing-review \& editing. FM provided substantial support in conceptualization, investigation, methodology, supervision, writing-original draft, writingreview \& editing. TN provided support in conceptualization, data curation, investigation, project administration, resources, visualization, writing-review \& editing. All authors contributed to the article and approved the submitted version.

\section{FUNDING}

DR-S was supported by the clinician scientists program Excellent Clinician Scientists in Freiburg - Education for Leadership (EXCEL) provided by the Else-Kroeger-Fresenius Forschungskolleg (grant number: 2020_EKFK.15) at the Medical Center - University of Freiburg, Faculty of Medicine, University of Freiburg, Germany.

\section{ACKNOWLEDGMENTS}

We would like to thank Jaqueline Landowski for excellent laboratory assistance.

\section{SUPPLEMENTARY MATERIAL}

The Supplementary Material for this article can be found online at: https://www.frontiersin.org/articles/10.3389/fonc.2021.658407/ full\#supplementary-material

proliferation. Br J Ophthalmol (2015) 99:943-8. doi: 10.1136/bjophthalmol2014-305893

7. Mets RB, Golchet P, Adamus G, Anitori R, Wilson D, Shaw J, et al. Bilateral Diffuse Uveal Melanocytic Proliferation With a Positive Ophthalmoscopic and Visual Response to Plasmapheresis. Arch Ophthalmol (2011) 129:1235-8. doi: 10.1001/archophthalmol.2011.277

8. Jaben EA, Pulido JS, Pittock S, Markovic S, Winters JL. The potential role of plasma exchange as a treatment for bilateral diffuse uveal melanocytic proliferation: a report of two cases. J Clin Apher (2011) 26:356-61. doi: $10.1002 /$ jca. 20310

9. Alrashidi S, Aziz AA, Krema H. Bilateral diffuse uveal melanocytic proliferation: a management dilemma. BMJ Case Rep (2014) 2014. doi: 10.1136/bcr-2014-204387

10. Hafer C, Golla P, Gericke M, Eden G, Beutel G, Schmidt JJ, et al. Membrane versus centrifuge-based therapeutic plasma exchange: a randomized prospective crossover study. Int Urol Nephrol (2016) 48:133-8. doi: 10.1007/ s11255-015-1137-3

11. Pulido JS, Flotte TJ, Raja H, Miles S, Winters JL, Niles R, et al. Dermal and conjunctival melanocytic proliferations in diffuse uveal melanocytic proliferation. Eye (Lond) (2013) 27:1058-62. doi: 10.1038/eye.2013.131 
12. Gass JD, Glatzer RJ. Acquired pigmentation simulating Peutz-Jeghers syndrome: initial manifestation of diffuse uveal melanocytic proliferation. Br J Ophthalmol (1991) 75:693-5. doi: 10.1136/bjo.75.11.693

13. Duong H-VQ, McLean IW, Beahm DE. Bilateral Diffuse Melanocytic Proliferation Associated With Ovarian Carcinoma and Metastatic Malignant Amelanotic Melanoma. Am J Ophthalmol (2006) 142:693-5. doi: 10.1016/j.ajo.2006.04.059

14. Prause JU, Jensen OA, Eisgart F, Hansen U, Kieffer M. Bilateral Diffuse Malignant Melanoma of the Uvea Associated with Large Cell Carcinoma, Giant Cell Type, of the Lung. OPH (1984) 189:221-8. doi: 10.1159/ 000309413
Conflict of Interest: The authors declare that the research was conducted in the absence of any commercial or financial relationships that could be construed as a potential conflict of interest.

Copyright (C) 2021 Rafei-Shamsabadi, Schneider, Trefzer, Technau-Hafsi, Meiss and Ness. This is an open-access article distributed under the terms of the Creative Commons Attribution License (CC BY). The use, distribution or reproduction in other forums is permitted, provided the original author(s) and the copyright owner(s) are credited and that the original publication in this journal is cited, in accordance with accepted academic practice. No use, distribution or reproduction is permitted which does not comply with these terms. 